\title{
College Students' Perceptions of the Safety of Marijuana and Cannabidiol Use While Breastfeeding
}

\author{
Erin M McKinley* \\ School of Nutrition \& Food Science, Louisiana State University, USA.
}

*Corresponding author: Erin M McKinley, School of Nutrition \& Food Science, Louisiana State University and the LSU AgCenter, Baton Rouge, LA, USA

\begin{abstract}
Use of cannabidiol (CBD), the non-psychoactive component, and delta-9-tetrahydrocannibinol (THC), the active psychogenic component in marijuana, is increasing in the United States as more states continue to legalize sales and consumption. As the public is becoming informed on the broad spectrum of therapeutic properties of these substances, including easing nausea and vomiting an increasing number of pregnant women are using marijuana to help with these issues. There is very little research on how people feel about cannabidiol product use during breastfeeding. The objective of this study was to assess college students' perceptions of marijuana product use during breastfeeding. A convenience sample of 675 college students completed a web-based survey that contained a valid general breastfeeding knowledge scale and questions to measure perceived level of agreement with the safety of marijuana products while breastfeeding. The mean breastfeeding knowledge score among this sample was low (179.84 \pm 24.47 ; range 96 - 246), with those scoring highest having the lowest perceived safety of marijuana use while breastfeeding. Significant differences in perceived safety were found based on gender $(p=0.036)$, race $(p=0.007)$, and personal breastfeeding experience $(p=.020)$. As new marijuana and breastfeeding research emerges, college health professionals should ensure that evidence-based information is used in family planning and infant feeding programs to educate college-age adults, who may become parents in the future. Future studies should include measures for personal cannabidiol product use and understanding and assess exposure to peers or mothers using marijuana products.
\end{abstract}

Keywords: Marijuana; Cbd; Breastfeeding; Lactation; Perceived Safety

\section{Introduction}

Use of cannabidiol (CBD), the non-psychoactive component, and delta-9-tetrahydrocannibinol (THC), the active psychogenic component in marijuana, is increasing in the United States (US) as more states continue to legalize sales and consumption [1]. As the public is becoming informed on the broad spectrum of therapeutic properties of these substances, including easing nausea and vomiting [2], an increasing number of pregnant women are using marijuana to help with these issues [3-5]. Use of these products are continuing after birth while mothers are actively breastfeeding. This study investigated college student's understanding and knowledge about breastfeeding and human lactation and their opinion regarding use of CBD and THC during breastfeeding. Published evidence on the effects of CBD itself on breastfeeding is scarce, but there are studies that have found that THC is transferred into breastmilk and may cause developmental delays in childhood $[6,7]$. With this information, medical professionals are advised to instruct pregnant and lactating women to discontinue use of any marijuana products during this time to avoid any possible negative effects [8-10]. Despite the recommendations, media portrayal of marijuana use does not reflect the same message. A 2018 review of online media content regarding marijuana use during pregnancy and the postpartum period found that only $51 \%$ of the content mentioned health risks of use, while $28 \%$ supported and promoted using marijuana for the treatment of nausea and vomiting during pregnancy [11]. There is also a shortage of public health messages about the risks of perinatal use of marijuana distributed at the state and federal level in the US [12]. Therefore, the public and current users of marijuana may still be ill informed of the negative consequences of use during pregnancy and lactation. One study found that $23.1 \%$ of US adults over the age of 18 surveyed felt that marijuana was "possibly unsafe" or "at least somewhat safe" during pregnancy [13]. College students are a subset of "emerging adults" in the US that are highly influenced by media and may be actively avoiding or engaging in social activities, such as using marijuana or other harmful substances, due to their perception of information from online and print sources [14]. This is also a population that 
may start seeking out health information related to family planning for future reference [15].

\section{Materials and Methods}

\section{Sample Population and Recruitment}

From February to April 2019, 675 participants were recruited at a public university in Louisiana where CBD and medical marijuana sales have been legalized. This study was approved by the LSU AgCenter Institutional Review Board prior to data collection. The investigator emailed a random selection of course instructors with the study details and requested a portion of class time to distribute the survey to students enrolled in their course. The investigator recruited participants in either the first or the last 10 minutes of class at the preference of the instructor. A slide with the shortened URL for the survey was projected on the screen in the classroom while the investigator described the details of the study. The informed consent was displayed on the first page of the web-based survey, created using Qualtrics ${ }^{\circledR}$ Online Surveys, which could be taken using a laptop or smartphone. Students who wanted to complete the survey could click "I Agree" to proceed to the next page of the survey. Anyone who clicked "I Disagree" was exited from the survey automatically. The survey took 8-12 minutes to complete and the investigator remained in the room to answer any participant questions. There was no incentive given to participants for completion of the survey.

\section{Materials}

An online platform-based survey contained a breastfeeding knowledge and understanding scale created for this study. It assessed participants' general understanding and knowledge about breastfeeding and human lactation. One item regarding safe use of CBD while breastfeeding and one item regarding discountenance of THC during breastfeeding were included. A demographics questionnaire assessed age, marital status, gender, race, if the participant was breastfed as a child, and if the participant had ever breastfed a child. Additional questions not relevant to this analysis were also included.

\section{Measures}

A breastfeeding knowledge scale was created for this study. The questionnaire assessed participants' general understanding of five areas of breastfeeding: benefits for the infant, the physical act of breastfeeding, common misconceptions, maternal benefits, and breastmilk safety. The scale consisted of 50 Likert-type questions, with answer choices of "strongly disagree, disagree, undecided, agree, and strongly agree." Seventeen items were reverse coded to reflect, "strongly disagree" as 5 decreasing in 1-unit increments to "strongly agree" as 1 . The remaining 33 items were coded as 1 for "strongly disagree" increasing in 1 -unit increments to 5 for "strongly agree." Higher scores indicate a greater amount of knowledge and understanding about breastfeeding. The 50-item scale had a Cronbach's alpha score of $\alpha=.944$ indicating strong internal consistency. The two items that assessed safety of CBD and discontinuance of THC while breastfeeding was written in the same fashion of the breastfeeding knowledge and understanding items but are not part of the 50 items on that scale. These two items were coded with a 1 for "strongly disagree" increasing in 1-unit increments to a 5 for "strongly agree." Because each measure consisted of only one item, reliability statistics were not computed.

\section{Data Analysis}

Descriptive statistics were analysed using Statistical Package for the Social Sciences (SPSS), version 25. Independent samples t-tests and one-way analysis of variance (ANOVAs), with Tukey post-hoc tests, assessed differences in perceived safety of THC and CBD while breastfeeding. Significance level was set at $\alpha<.05$.

\section{Results}

The sample included 486 (72\%) females and 155 (23\%) males who were $77 \%$ white, $10.4 \%$ black, $6.2 \%$ Asian, and $6.4 \%$ "other" races. The students had a mean (SD) general breastfeeding knowledge scale score of 179.84 (24.47) with a range of 96 to 246 indicating a relatively low level of knowledge and understanding of breastfeeding and lactation.

\section{Perceived safety of marijuana}

The sample's overall mean (SD) response to the item asking about use of THC while breastfeeding was 4.09 (.989), indicating moderate agreement with the idea that a mother who plans to breastfeed should refrain or significantly reduce usage. Seventyfive percent $(75.1 \%)$ of the sample either agreed or strongly agreed with this idea. There was a significant difference in opinion between males and females on this issue $(p=.008)$, with females having stronger agreement with abstaining from THC. Black students had significantly higher agreement with reducing or refraining from THC use than white students did ( $\mathrm{p}=.041)$. Students in the sample who had previously breastfed a child (or whose partner had breastfed their child) had significantly lower agreement with avoiding THC $(p=.020)$ than those with no breastfeeding experience. Those who did not know if they were breastfed as children or not had significantly less agreement than the other students regarding non-use of THC ( $p=.002)$. A significant difference between opinion on THC use while breastfeeding and mean general breastfeeding knowledge was found $(p=.027)$. Those students with the strongest disagreement with abstaining from THC while breastfeeding had the highest mean breastfeeding knowledge score. The students who were undecided on the issue had significantly lower mean breastfeeding knowledge scores than those with the strongest agreement to not use THC while breastfeeding ( $p=.036)$. These differences are displayed in Table 1.

\section{Perceived safety of cannabidiol}

The study sample's overall mean (SD) response to the item asking about the safe use of CBD while breastfeeding was 2.92 (1.10), indicating moderate disagreement with the idea that a mother can 
safely use CBD products while breastfeeding. Twenty-six percent $(26.2 \%)$ of the sample either disagreed or strongly disagreed with the idea, while $42.4 \%$ were undecided on the issue. Females had significantly stronger disagreement with CBD safety than the male students did $(p=.036)$. Black students had the strongest disagreement with safe use of CBD that was significantly stronger ( $p=.007)$ than white students and those of other races (American
Indian or Alaska Native, Native Hawaiian or other Pacific Islander). The students with the strongest agreement with safe use of CBD products while breastfeeding had the highest mean breastfeeding knowledge scores. Those with the strongest agreement with safe use had significantly higher mean knowledge scores than those who were undecided or in slight disagreement with the issue ( $p$ $<.001)$. These results are displayed in Table 1.

Table 1: Differences in perception of safe of THC and CBD by demographics.

\begin{tabular}{|c|c|c|c|c|c|c|}
\hline & $\mathbf{n}$ & $\%$ & $\begin{array}{l}\text { "Mothers who use marijuana THC should } \\
\text { stop using or significantly reduce usage if } \\
\text { they plan to breastfeed their baby." Mean } \\
\text { (SD) }\end{array}$ & P-value & $\begin{array}{l}\text { “Mothers can safely use legal } \\
\text { cannabidiol (CBD) products and } \\
\text { breastfeed their child." Mean } \\
\text { (SD) }\end{array}$ & P-value \\
\hline Age & 646 & & & 0.13 & & 0.165 \\
\hline $18-20$ & 292 & 45.2 & $4.08(1.01)$ & & $2.88(1.12)$ & \\
\hline $21-26$ & 219 & 40 & $4.21(.936)$ & & $2.87(1.11)$ & \\
\hline $27-76$ & 135 & 20.8 & $4.01(.962)$ & & 3.08 (1.07) & \\
\hline Marital Status & 650 & & & 0.209 & & 0.075 \\
\hline Single & 546 & 84 & $4.12(.977)$ & & $2.88(1.10)$ & \\
\hline Married & 104 & 16 & $4.00(1.00)$ & & $3.13(1.14)$ & \\
\hline Gender & 649 & & & $.008^{*}$ & & $.036^{*}$ \\
\hline Male & 155 & 23.8 & $3.94(1.10)$ & & $3.02(1.08)$ & \\
\hline Female & 486 & 74.8 & $4.17(.919)$ & & $2.87(1.11)$ & \\
\hline Othera & 8 & 1.4 & $3.5(1.69)$ & & $3.75(1.16)$ & \\
\hline Race & 644 & & & $.041^{*}$ & & $.007^{*}$ \\
\hline White & 496 & 77 & $4.05(.976)$ & & $2.98(1.10)$ & \\
\hline Black & 67 & 10.4 & $4.42(.873)$ & & $2.54(1.11)$ & \\
\hline Asian & 40 & 6.2 & $4.08(.971)$ & & $2.70(.966)$ & \\
\hline Otherb & 41 & 6.4 & $4.12(1.20)$ & & $3.12(1.24)$ & \\
\hline Breastfed as Child & 647 & & & $.002 *$ & & 0.912 \\
\hline Yes & 419 & 64.7 & $4.14(.952)$ & & $2.92(1.13)$ & \\
\hline No & 142 & 21.9 & $4.20(.919)$ & & $2.88(1.11)$ & \\
\hline Don't Know & 86 & 13.4 & $3.77(1.11)$ & & $2.94(1.10)$ & \\
\hline Ever Breastfed Child & 499 & & & $.020^{*}$ & & 0.096 \\
\hline Yes & 86 & 17.3 & $3.88(1.01)$ & & $3.09(1.15)$ & \\
\hline No & 413 & 82.7 & $4.14(.949)$ & & $2.85(1.08)$ & \\
\hline \multicolumn{7}{|c|}{ NOTE: *Significant at a p-value $<.05$} \\
\hline
\end{tabular}

\section{Discussion}

With an increasing number of states legalizing sales of marijuana and CBD products, it is important to look at how the public perceives the safety of these products. It is especially important to gauge perceptions of safety among populations that may experience deleterious effects, including pregnant and breastfeeding women. College students are a vital population to explore this as they may be actively exploring the use of marijuana, as well as, learning and preparing for possible major life events such as pregnancy and parenthood. One previous studying evaluating adult's perceptions of safety of marijuana while pregnant showed that although most of the sample agreed it was completely unsafe, there was still one fourth of the sample that was unsure or thought it may be somewhat safe [13]. Previous literature has also shown that media and public health messages in the US have displayed mixed messages regarding safety of marijuana during pregnancy 
and the postpartum period [11,12]. The current work expands on previous research by reaching into the perceptions of adults in college on the safety of marijuana during the specific postpartum act of breastfeeding. The research regarding THC exposure through breastfeeding causing possible negative effects to a child is available, but CBD safety in breastfeeding is not readily available, so asking separate questions about these two substances was important in this study. Despite separating CBD from THC in the survey questions, this sample of college students looked at both forms of marijuana as moderately unsafe while breastfeeding, with CBD viewed as slightly less unsafe. This may be due to the current surge in media focus on CBD with the passing of the 2018 Farm Bill [16] including hemp as a commercial agriculture product in the US. This in turn legalized hemp (CBD) products at a federal level opening the doors to production and sale of the product across the nation. At the time of this study, previously legalized medical marijuana became available to prescribers in the state and the state legislature voted to approve hemp and CBD sales.

\section{Implications}

An increase in general knowledge about breastfeeding and lactation does not necessarily equate to increased knowledge, understanding, and perception that THC and CBD may not be safe for breastfeeding mothers to consume. Either this finding implies that college-age adults who are knowledgeable about general information on breastfeeding have not been informed about the consequences of potentially harmful substances, or the media "buzz" surrounding these products in the weeks surrounding this study influenced participants' responses. As published research articles regarding effects of THC and CBD start to increase in number, along with the public's growing curiosity to know the truth, educational efforts need to also increase content and messages regarding marijuana and breastfeeding. The new literature on benefits and consequences of marijuana may continue to conflict, but health educators need to air on the side of safety and inform and educate appropriately. Future studies should include additional measures for personal marijuana product use and understanding, exposure to mothers using marijuana products, and focus on students planning to go into family and consumer science, health, wellness, or medical career fields. Additionally, future studies should aim to replicate this study in a population of adults outside that of a college or university setting from more diverse socioeconomic, educational, racial, and ethnic backgrounds.

\section{Limitations}

There were several limitations to this study. First, the study had a relatively small sample of students, who were majority white and female (55\%), at one university in the southern United States. The results may not generalize to all students at this university, all college students in states with legal CBD and marijuana availability, and all college students across the United States. Second, the study did not ask students about their personal use of CBD and marijuana containing THC or the perceived safety of these substances in any other regard than the two survey items about breastfeeding. Finally, the survey in its entirety was 78 questions, which may have caused participant fatigue or instances of rushing to get it done before getting to leave class, possibly effecting how students were answering questions. No attention check questions were used in the survey to flag participants who may not have been reading items carefully.

\section{Acknowledgements}

The author would like to thank Hilary Carrera Arcia for her assistance in data collection and overall study management.

\section{Conflict of Interest Disclosure}

This material is based upon work that is supported by the National Institute of Food and Agriculture, U.S. Department of Agriculture, Hatch project under LAB94426.

\section{Reference}

1. Metz TD, Stickrath EH (2015) Marijuana use in pregnancy and lactation: a review of the evidence. Am J Obstet Gynecol 213(6): 761-778.

2. Mannucci C, Navarra M, Calapai F, Spagnolo EV, Busardò FP, et al. (2017) Neurological aspects of medical use of cannabidiol. CNS Neurol Disord Drug Targets 16(5): 541-553.

3. Brown QL, Sarvet AL, Shmulewitz D, Martins SS, Wall MM, et al. (2017) Trends in marijuana use among pregnant and nonpregnant reproductive-aged women, 2002-2014. JAMA 317(2): 207-209.

4. Young Wolff KC, Sarovar V, Tucker LY, Avalos LA, Alexeeff S, et al. (2019) Trends in marijuana use among pregnant women with and without nausea and vomiting in pregnancy, 2009-2016. Drug Alcohol Depend 196: 66-70.

5. Young Wolff KC, Tucker LY, Alexeeff S, Armstrong, MA, Conway A, et al. (2017) Trends in self-reported and biochemically tested marijuana use among pregnant females in California from 2009-2016. JAMA 318(24): 2490-2491.

6. Djulus J, Moretti M, Koren G (2005) Marijuana use and breastfeeding. Can Fam Physician 51(3): 349-350.

7. Murphy LL, Muñoz RM, Adrian BA, Villanúa MA (1998) Function of cannabinoid receptors in the neuroendocrine regulation of hormone secretion. Neurobiol Dis 5(6): 432-446.

8. Braillon A, Bewley S (2018) Committee opinion no. 722: marijuana use during pregnancy and lactation. Obstet Gynecol 131(1): 164.

9. Ryan SA, Ammerman SD, O Connor ME (2018) Marijuana use during pregnancy and breastfeeding: implications for neonatal and childhood outcomes. Pediatrics 142(3): e20181889.

10. Seabrook JA, Biden C, Campbell E (2017) Does the risk of exposure to marijuana outweigh the benefits of breastfeeding? A systematic review. Canadian Journal of Midwifery Research and Practice 16(2): 8-16.

11. Jarlenski M, Koma JW, Zank J, Bodnar LM, Tarr JA, et al. (2018) Media portrayal of prenatal and postpartum marijuana use in an era of scientific uncertainty. Drug Alcohol Depend 187: 116-122.

12. Jarlenski M, Zank J, Tarr J, Chang JC (2017) Public health messages about perinatal marijuana use in an evolving policy context. Subst Abus 38(1): 48-54.

13. Keyhani S, Steigerwald S, Ishida J, Vali M, Cerdá M, et al. (2018) Risks and benefits of marijuana use: A national survey of US adults. Ann Intern Med169(5): 282-290. 
14. Kim Y, Wang Y, Oh J (2016) Digital media use and social engagement: How social media and smartphone use influence social activities of college students. Cyberpsychol Behav Soc Netw 19(4): 264-269.

15. Rennis L, McNamara G, Seidel E, Shneyderman Y (2015) Google it!: urban

To Submit Your Article Click Here:

This work is licensed under Creative

Commons Attribution 4.0 License

DOI: $10.32474 /$ SJFN.2021.03.000175

community college students' use of the internet to obtain self-care and personal health information. College Student Journal 49(3): 414-426.

16. United States Senate Committee on Agriculture, Nutrition, and Forestry. (2018) 2018 Farm Bill.

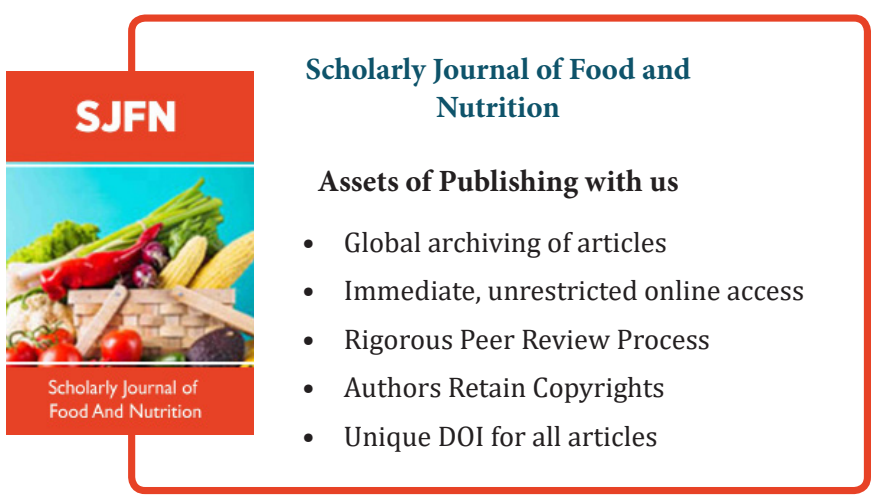

